\title{
La especialización del saber en la escuela: intervenciones estatales en campos específicos de conocimiento
}

\section{Resumen}

Cecilia Acevedo ${ }^{1}$

Este trabajo se propone analizar la implementación de programas educativos específicamente orientados al campo de conocimiento de las Ciencias Naturales en escuelas primarias de la ciudad de Buenos Aires. Con los programas, ingresan a las escuelas discursos y prácticas a través de personas que se incorporan a trabajar con nuevas funciones docentes y que cuentan con mayores grados de especialización que los maestros de grado. Se aborda cómo incide la penetración de esta especialización en la vida escolar y, en particular, los conflictos que conlleva la incorporación de docentes especialistas. En el marco de una investigación de enfoque etnográfico, la descripción del entramado de relaciones entre todas las personas encargadas de la enseñanza, la consideración de sus perspectivas y las significaciones otorgadas a diferentes dimensiones que forman parte de la lógica práctica del orden escolar, condujeron a colocar el foco de análisis en la irrupción de las posiciones históricas vinculadas al saber. Como efecto de esta conmoción de la vida cotidiana escolar, se produce un (re)ordenamiento basado en un proceso de diferenciación que termina por instalar la idea de que los maestros ya no portan los saberes que históricamente legitimaban su posición. Se desdibuja su autoridad pedagógica, cuestión que afecta y modula los vínculos que se establecen con los saberes de un campo de conocimiento en la escuela.

\section{Palabras clave}

Programas educativos - Conocimiento - Especialización - Enseñanza de las ciencias - Etnografía.

\section{Knowledge specialization in school: state intervention in specific fields of knowledge}

\section{Abstract}

The purpose of this paper is to analyze how specific educational programs related to the knowledge of Natural Sciences are implemented in Buenos Aires elementary schools. Programs, together with new speeches and practices, are introduced by people with new

1- Universidad de Buenos Aires, Buenos Aires, Argentina. Contacto: Iceciliaacevedo@gmail.com

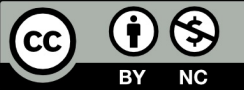

DOl: http://dx.doi.org/10.1590/S1678-4634201844175336

This content is licensed under a Creative Commons attribution-type BY-NC. 
teaching functions and higher degrees of specialization than current classroom teachers. This study deals with how the penetration of this specialization affects school life and focuses on the conflicts involved in the incorporation of specialist teachers. These findings are part of an ethnographic approach research. The description is based on the network of relationships among all the people in charge of teaching, the consideration of their perspectives and the meanings given to different dimensions that form part of the practical logic of school order. These led to place the focus of analysis on the irruption caused on the historical positions linked to knowledge. As a result of this commotion of everyday school life, there is a reordering based on a process of differentiation that ends up installing the idea that teachers no longer carry the knowledge that historically legitimized their position. Its pedagogical authority is blurred. This issue affects and modulates the links established with knowledge in school.

\section{Keywords}

Educational programs - Knowledge - Specialization - Science teaching - Ethnography.

\section{Presentación}

La enseñanza y el aprendizaje de las Ciencias Naturales han ocupado tanto al campo de investigaciones de la didáctica específica como al de las políticas educativas. En los últimos años, una de las facetas de estas políticas ha sido la intervención estatal a través de programas educativos.

Desde los orígenes del sistema educativo, los maestros de grado estuvieron a cargo de la enseñanza de las ciencias en la escuela. En la actualidad, hay otras personas que ingresan a través de los programas -en calidad de maestros especializados, asistentes técnicos, capacitadores. Cuando llegué a las escuelas donde realicé el trabajo de campo, todas estas personas encargadas de la enseñanza del área insistieron en verse diferentes. Los maestros de grado decían que lo de ellos era otra cosa. A la par, esas otras personas insistían que lo suyo se distanciaba de la enseñanza común. En este trabajo me pregunto qué efectos produce en la vida escolar el ingreso de personas que trabajan bajo la órbita de los programas.

Desde la didáctica específica se han producido diferentes herramientas analíticas con vistas a comprender, explicar y favorecer los procesos de enseñanza y aprendizaje de las Ciencias Naturales. Entre ellas, se ha avanzado en conocer las concepciones más recurrentes de los estudiantes sobre determinados saberes disciplinares -campo generalmente conocido como ideas previas-, en caracterizar la especificidad de los contenidos y los conceptos estructurantes del área, en estudiar la relación de los profesores con tales saberes y sus concepciones epistemológicas, así como en analizar las interacciones que se producen en clase a propósito del conocimiento. 
En este trabajo ${ }^{2}$ el análisis estará centrado en la vida cotidiana escolar y en los significados que diferentes actores -no solo los docentes que llevan adelante una propuesta didáctica y los alumnos a quienes está destinada- les otorgan a diferentes dimensiones de esta cotidianeidad. Partiendo de que la relación con el saber es la relación con uno mismo y con los otros (CHARLOT, 2007), me propongo mostrar que esta relación se encuentra condicionada por los modos de decir y estar en las escuelas, por las posiciones que los actores ocupan dentro de un entramado de relaciones en los cuales se incluye a otros docentes, niños, padres, recursos, tiempos y espacios para el desarrollo de sus trabajos, modos de ingreso, escalafones y denominación de sus puestos de trabajo. Sostengo que todo esto no puede explicarse sin atender las transformaciones estatales.

Como efecto, se produce un (re)ordenamiento escolar basado en un proceso de diferenciación donde se dirimen los posicionamientos relativos al saber. En este (re) ordenamiento termina por instalarse la idea de que los maestros de grado ya no portan los saberes que antaño legitimaban su posición histórica en las escuelas -cuestión que afecta la relación que se entabla con el conocimiento y que resulta de difícil anticipación desde el diseño de las políticas educativas.

Estos hallazgos forman parte de una investigación etnográfica que se interesa por los modos de presencia estatal y sus efectos en los procesos de enseñanza y aprendizaje de las Ciencias Naturales en escuelas primarias de la ciudad de Buenos Aires -Argentina- que cuentan con programas especialmente orientados a este dominio de conocimiento.

\section{Orientaciones conceptuales}

Consideré a los programas como políticas educativas en las cuales la intervención del Estado no se limita a las instituciones y a las burocracias, sino que puede localizarse en prácticas y procesos que producen determinados efectos en los sujetos (TROUILLOT, 2001). En esta línea, los aportes de la etnografía educativa me han permitido considerar a la institución escolar como ámbito en el cual es posible estudiar la configuración misma del Estado, sus transformaciones y sus presencias -en tanto objeto analítico- (MILSTEIN, 2009; ROCKWELL, 2009).

Comprender a la escuela a partir de la lógica práctica inscripta en los sujetos de la enseñanza y del aprendizaje, me permitió visibilizar cómo lo actuado en situaciones de la vida cotidiana escolar entraba en fricción con el ingreso de nuevos actores estatales en las escuelas.

La lógica práctica es una categoría del pensamiento de Bourdieu (2007) con la cual se refiere a aquello que los sujetos actúan, deciden en acto, en función de sus posibilidades objetivas. De los trabajos de Milstein y Mendes (1999, 2013) he considerado a la lógica práctica del orden escolar como las reglas implícitas que regulan los modos de decir, estar y moverse en las escuelas que pasan a formar parte de lo que la escuela es.

2- Este trabajo presenta parte de los resultados de una investigación doctoral desarrollada y defendida en la Universidad Nacional de Quilmes. La autora ha recibido dos becas financiadas por el Ministerio de Ciencia, Tecnología e Innovación Productiva de la Nación y por el CONICET. 
El clásico trabajo de Rockwell (1989) me permitió identificar una serie de dimensiones -tiempos, espacios y estructuras de participación- que pautan relaciones sociales, regulan las interacciones, a la par que dichas pautas se proyectan en el entorno social amplificando la experiencia formativa dentro de la institución. He interpretado estos aportes en una relación donde las condiciones objetivas como las percepciones y disposiciones a pensar, decir y actuar se constituyen mutuamente y no están dadas de una vez para siempre.

Por último, consideré el concepto de autoridad pedagógica. Según Bourdieu y Passeron (1998) todo poder involucra relaciones de dominación que se producen al interior de las instituciones en las cuales se imponen significados vinculados a cierta cultura dominante -un arbitrario cultural- que se presentan como legítimos. Dicha imposición se realiza bajo el disimulo de las relaciones de fuerza, lo que contribuye a la reproducción de estas relaciones y de los significados inculcados. Desde esta perspectiva, la acción pedagógica inculca una selección arbitraria de significados pertenecientes a un grupo.

Quienes se constituyen en receptores del mensaje pedagógico están dispuestos a interiorizar el mensaje inculcado por el solo hecho de ser transmitido por emisores que gozan de autoridad. El concepto de autoridad pedagógica -que se enmarca dentro de la teoría de la violencia simbólica- es explicado en relación con un espacio social de luchas simbólicas por la desigual distribución de los bienes culturales (BOURDIEU, 1990). Se produce así un espacio de relaciones de poder simbólico en donde los agentes pertenecientes a ese espacio adquieren posiciones relativas en función de la cantidad y la calidad de las distintas especies de capital que poseen.

Estos aportes me permitieron situar el carácter relativo de las posiciones de los encargados de la enseñanza, materiales, tiempos, espacios y jerarquías que se internalizan encarnándose en los sujetos. Esta internalización supone el disimulo de las relaciones de fuerza, una eficacia simbólica, que conlleva cierto olvido de la génesis de dichas relaciones (BOURDIEU, 2012). Ello no significa la inadvertencia de las personas respecto de los malestares, tensiones, significaciones que se despliegan a su alrededor sino una suerte de inercia en la cual se inscriben las actuaciones. En este sentido interpreté que el ejercicio de la violencia simbólica es invisible, huidizo, a los actores.

\section{El trabajo etnográfico}

Asumí que la singularidad del trabajo etnográfico reside en la empresa involucrada en la elaboración del conocimiento en su triple acepción de enfoque, método y texto (GUBER, 2008). Desde la tradición antropológica, implica un aporte epistemológico para la investigación social sobre el conocimiento de la otredad. Desde lo teórico-metodológico, se trata de un abordaje donde cobra relevancia el concepto de reflexividad. Y, desde el texto etnográfico, se concibe la singularidad de la descripción en la medida que involucra la interpretación del investigador sobre la de los informantes.

El trabajo de campo lo realicé a lo largo de 2009. En principio, supuso visitas semanales que me permitieron contactarme con maestros, asistentes, capacitadores, 
directivos de cada una de las escuelas. Luego presencié el desarrollo de propuestas de enseñanza. Este período involucró visitas de hasta tres veces semanales en cada una de las escuelas. Registré conversaciones informales, realicé observaciones y entrevistas.

Las instancias de análisis las produje a lo largo de todo el proceso de investigación incluyendo la alternancia de momentos de escritura -elaboración de registros ampliados, del diario de campo y de redacción de descripciones-, reescritura, lectura y relectura sistemática. Fue a través de poner en relación palabras, acciones y significados, tanto de los actores como propias, que pude densificar las descripciones (GEERTZ, 1992) y acceder a la elaboración conceptual de nuevas relaciones en las que se "[...] puede dar cuenta mejor del orden particular, local y complejo del proceso estudiado" (ROCKWELL, 2009, p. 67).

\section{El escenario}

El proceso de recuperación económica de mediados de 2000 vino acompañado de un redireccionamiento de las políticas públicas que buscaban diferenciarse de la consolidación del neoliberalismo de la década anterior. En este marco, el área de las Ciencias Naturales -junto con la ciencia y la tecnología- ha cobrado interés y ha sido objeto de programas de orden nacional y jurisdiccional. La convivencia de programas nacionales y jurisdiccionales dentro de una misma jurisdicción es consecuencia de las transformaciones en la normativa que rigió al sistema educativo a partir de su descentralización completada durante los años noventa. El Ministerio de Educación Nacional -sin escuelas a cargo- propicia programas que requieren la intervención de las jurisdicciones para su implementación. A la par, cada jurisdicción tiene programas propios que se desenvuelven conforme a las leyes marco que rigen para todo el sistema educativo nacional.

Los programas suelen iniciarse a partir del diagnóstico de algún aspecto o área de vacancia que merezca atención. Su funcionamiento se rige bajo la creación de nuevas dependencias estatales cuyo financiamiento puede provenir de una nueva distribución del presupuesto nacional, jurisdiccional ${ }^{3}$ o de organismos internacionales.

Los programas se diferencian de otras dependencias estatales por atender asuntos particularizados y por su mayor grado de incertidumbre respecto de su permanencia en el tiempo, el financiamiento, y la continuidad laboral de sus trabajadores.

El funcionamiento de los programas en las escuelas involucra el ingreso de personas, como asistentes técnicos o capacitadores, a partir de mecanismos diferentes a los que rigen para el resto de las personas que ya trabajan dentro de éstas. Aparecen nuevos espacios de trabajo, materiales didácticos, planificaciones, libros, laboratorios. A su vez, por fuera de las escuelas, se instalan nuevas coordinaciones, espacios de formación, muestras de ciencias, requerimientos administrativos como la elaboración de informes.

3- La ciudad de Buenos Aires es considerada una de las veinticuatro jurisdicciones que integran el territorio nacional. 
Las normativas que rigen el funcionamiento de los programas pueden vincularse a lo que Giovine (2012) denomina legislación derivada. Con esta categoría, remite a la proliferación de resoluciones, programas, proyectos e informes en la cual se multiplican los centros de regulación estatal. Según la autora, las reformas en la legislación producidas en los años noventa -período que involucró la descentralización del sistema educativo nacional- devino en la convivencia de viejas y nuevas formas de regulación, control y decisión más locales.

A continuación, presento a las escuelas estudiadas junto a los programas que funcionaban dentro de éstas y a las personas con los cuales interactué más asiduamente.

\section{La escuela $\mathrm{n}^{\circ} 8$}

La escuela primaria $n^{\circ} 8$ se ubica al oeste del centro de la ciudad. Tiene dos jornadas simples - mañana y tarde- con dos secciones por grado -A y B. Recibe cerca de cuatrocientos alumnos. Los miércoles, en ambos turnos, funciona un programa denominado Club de Ciencias.

Si bien los Clubes de Ciencias fueron creados a mediados de los años ochenta, en esta escuela comenzó a funcionar en 2007. Se trata de una propuesta extracurricular orientada a niños y/o jóvenes que presenten interés en profundizar contenidos de las Ciencias Naturales. Se desarrollan en los laboratorios de escuelas sede dispuestas a recibir a estudiantes de la propia institución o de otras escuelas del distrito. Su horario se dispone a contra turno en las escuelas de jornada simple y como jornada extendida en escuelas de jornada completa.

Ana era la docente de Matemática y Ciencias Naturales de séptimo grado del turno mañana. Luciano era el maestro del Club de Ciencias. Al igual que Ana, también era maestro de grado, pero para aquel entonces no ejercía como tal.

\section{La escuela $n^{\circ} 20$}

La escuela primaria $n^{\circ} 20$ pertenece a un distrito escolar de la zona sur de la ciudad. Es de jornada completa y tiene dos secciones con poco más de veinte chicos por curso.

En esta escuela funcionaban dos programas: Intensificación en Actividades Científicas (IAC) y el Programa Integral para la Igualdad Educativa (PIIE).

El primero de ellos se creó en 2004. La propuesta presenta mayor desarrollo curricular en Ciencias Naturales ofreciendo una profundización en los contenidos. Para la escuela significaba contar con la presencia de dos asistentes técnicos, uno por cada ciclo, y un capacitador que daba clases quincenales a los maestros de cada ciclo sobre contenidos de ciencias.

El segundo programa, PIIE, consiste en financiamiento destinado al mejoramiento de la enseñanza y el aprendizaje. Con el dinero recibido se compran materiales y afrontan los gastos relativos a actividades y proyectos previamente acordados con los asistentes técnico-pedagógicos del programa. Durante el período que realicé el trabajo de campo, no se había designado un asistente técnico para esta escuela. 
Claudio era el maestro de grado de Prácticas del Lenguaje y de Ciencias Naturales de séptimo grado. Dante era profesor de Biología y trabajaba en la escuela como asistente técnico de IAC para el segundo ciclo.

\section{Ingresos y escalafones diferenciados}

En la ciudad de Buenos Aires, el área de nivel primario y los programas ${ }^{4}$ conforman dependencias administrativas que pertenecen a la misma jurisdicción estatal y se diferencian entre sí por "su carácter educativo específico" (BUENOS AIRES, 2014, p. 1). Como mencioné más arriba, los maestros de grado y las personas que trabajan en los programas ingresan a las escuelas por mecanismos diferenciados.

Los primeros deben inscribirse en las juntas de clasificación docente, exhibir los cursos realizados, participar de la asignación de puntajes por su formación y antigüedad docente y presentarse a los actos públicos. Para tomar un cargo, titularizarlo y ascender de escalafón, los maestros de grado, Ana, Luciano y Claudio lo hacían dentro del mecanismo histórico expresado en el Estatuto del Docente.

Para los segundos, las vías administrativas presentaban variaciones entre y al interior de cada programa. Luciano, el maestro de Club de Ciencias, ejercía como maestro de grado cuando se enteró de la existencia del programa. Esperó que se abriera una convocatoria; presentó una propuesta de enseñanza; la defendió en un coloquio; y, más allá de su antigüedad y de los cursos realizados durante su formación, ingresó a trabajar en el programa entrando en comisión de servicios ${ }^{5}$. Este hecho hizo que, más adelante, pudiera ascender de escalafón al presentarse en un nuevo acto público -regido por los listados donde se inscriben los maestros de grado- y tomar un cargo directivo.

Para ingresar en IAC, Dante fue recomendado por una compañera. Se presentó a una entrevista y, sin contar con el título docente requerido históricamente para ejercer la docencia en el nivel primario, fue contratado.

Con los programas se desregularizan los modos históricos para el ingreso y el ejercicio de la docencia. Resulta sumamente novedoso, por su carácter reciente, que en las escuelas ingresen a trabajar docentes por fuera de los mecanismos habituales que hace muchos años se instauró. Como puede apreciarse en la tabla 1, el área de Programas Socioeducativos y el área de la Educación Primaria cuentan, a su vez, con una serie escalafonaria propia.

\footnotetext{
4- En 2010, diferentes programas que funcionaban en la ciudad de Buenos Aires fueron aglutinados bajo una misma estructura denominada Programas Socioeducativos. A fines de ese año, el Gobierno sancionó la ley n 3623 en la cual esta estructura era reconocida dentro del Estatuto del Docente.

5- Se refiere a la situación en la que un docente realiza una tarea pedagógica diferente por la cual asumió su cargo titular, ya sea en otra área o en actividades gremiales, y en la que no pierde su cargo inicial por la realización de esta nueva tarea.
} 
Tabla 1- Escalafón docente, según área educativa.

\begin{tabular}{|c|c|c|}
\hline \multicolumn{2}{|c|}{ Área educativa } & Escalafón docente \\
\hline \multicolumn{2}{|c|}{ Educación primaria } & $\begin{array}{l}\text { a) Maestro de grado } \\
\text { b) Maestro secretario } \\
\text { c) Vicedirector } \\
\text { d) Director } \\
\text { e) Supervisor Adjunto de Educación Primaria } \\
\text { f) Supervisor de Educación Primaria } \\
\text { g) Director Adjunto de Educación Primaria } \\
2- \\
\text { a) Asistente de Comedor }\end{array}$ \\
\hline \multirow[t]{2}{*}{ Programas socioeducativos } & Club de Ciencias & $\begin{array}{c}\text { Maestro/a de Programa / Profesor/a. } \\
\text { Coordinador/a Pedagógico/a. } \\
\text { Coordinador/a de Programa. } \\
\text { Coordinador/a. }\end{array}$ \\
\hline & $\begin{array}{c}\text { Intensificación en } \\
\text { Actividades Científicas (IAC) }\end{array}$ & $\begin{array}{c}1 \text { - } \\
\text { Asistente Técnico/a pedagógico/a. } \\
\text { Coordinador/a General. } \\
\text { 2- No escalafonado/a: } \\
\text { Capacitador/a. } \\
\text { Auxiliar operativo/a. } \\
\text { Asistente. }\end{array}$ \\
\hline
\end{tabular}

Fuente: Elaborado para este estudio en base al Estatuto del Docente (BUENOS AIRES, 2014).

La convivencia de vías de ingreso y escalafonamiento configuradas históricamente con nuevas modalidades instaladas a partir de los programas es avalada por el mismo Estado. En las modalidades de trabajo configuradas históricamente, las relaciones de paridad, jerarquía y ascenso de escalafón estaban claramente pautados. En los programas, si bien hay delimitados escalafones, no hay mecanismos explicitados respecto de cómo se asciende, ni claridad sobre las relaciones de paridad y jerarquía dentro de las escuelas. A pesar de estas diferencias, la normativa considera que todas estas personas son docentes (BUENOS AIRES, 2014). Sin embargo, como mostraré a continuación, no todas son así consideradas dentro de las escuelas. Ser considerado docente y las funciones que cada una desempeña son categorías que se dirimen en la vida cotidiana escolar.

\section{Puestos de trabajo en la cotidianeidad escolar}

En las escuelas estudiadas, los maestros y las personas que ingresan a través de los programas no se reconocían a sí mismos en términos de un nosotros que podría referir a un colectivo cuya función es ocuparse de la enseñanza de las ciencias. Por el contrario, se producian diferentes categorias discursivas que introducían una diferenciación respecto 
de un otro que ocupa y ejerce la docencia en diferentes tiempos y espacios dentro de la misma institución escolar.

Iniciando el trabajo de campo, tuve reuniones con maestros de grado e integrantes de los equipos directivos. En ambas escuelas, comenté que tenía interés en estudiar los procesos de enseñanza y aprendizaje de las ciencias en la vida cotidiana de la escuela.

Frente a esta presentación, la directora de la escuela $n^{\circ} 8$ mencionó que “[...] los maestros acuden mucho al profe del Club de Ciencias para hacerle consultas sobre posibles experimentos", que era un profe especializado 6 . Ana, la maestra de séptimo grado, que durante los diez primeros minutos de la conversación prácticamente no había hablado, me pidió inmediatamente que por favor no la comparara con Luciano "porque nada que ver” (Notas de campo, 18/06/2009). Más tarde cuando conocí a Luciano noté, en cambio, que se refería a sí mismo o bien como maestro de grado o bien como maestro del Club de Ciencias $^{7}$. Si bien Luciano también era maestro de grado, para la directora era un profe especializado, para Ana era el profesor de ciencias.

El puesto de Luciano era referido dentro de la escuela en base a su especialización en un campo de conocimiento mientras que la normativa lo hacía por su inscripción al programa. El puesto de Ana prácticamente no sufría variaciones en los modos de ser nombrado. Para los niños, tanto Luciano como Ana eran simplemente profes.

En el primer encuentro en la escuela $n^{\circ} 20$, la secretaria no dudó en sugerirme que debía participar de las clases de tercer grado porque el docente era ingeniero agrónomo. Al igual que en la escuela anterior, los puestos de trabajo también gozaban de diferentes denominaciones connotadas por diferentes grados de especialización en las Ciencias Naturales.

Cuando los maestros presentaban algún estudio -concluido o en curso- afín al dominio de conocimiento en cuestión, los integrantes del equipo de conducción se referían a ellos a través de esta titulación. Éste era el caso del maestro que estudiaba ingeniería agrónoma y que aún no era ingeniero agrónomo como lo presentaba la secretaria. Los maestros que no poseían estos estudios eran referidos como maestros por los demás encargados de la enseñanza. Inclusive, el modificador indirecto que acompañaba maestro de se refería al grado de escolarización y no a las asignaturas de las cuales se encargaba. Es decir, Claudio, el maestro de séptimo, era también el maestro de Prácticas del Lenguaje y de Ciencias Naturales.

El puesto de Dante, el asistente técnico del programa, era el que más variaciones de nombres recibía, contando las alusiones que él realizaba de sí mismo recurriendo a otras inscripciones institucionales tales como profesor de media ${ }^{8}$, investigador o capacitador. En ocasiones, Dante decía que parecía un extra curricular porque llegaba un punto en donde si el docente no estaba metido en el tema y no asumía el proyecto como suyo, dejaba de ser el asistente técnico para ser el profesor de IAC, que es como lo conocían los chicos. Claudio, en cambio, lo veía como un capacitador pero no como un profesor, “[...] habrá

\footnotetext{
6- En el texto utilizo la cursiva para dar cuenta de las categorías nativas que pertenecen a los discursos de las personas con las cuales pude dialogar durante el trabajo de campo, al campo de la didáctica específica, a los organismos de gobierno y a los organismos internacionales.

7- No todas las personas que ingresaban a trabajar en Club de Ciencias eran maestros, algunos eran profesores de nivel terciario o universitario en disciplinas afines a las Ciencias Naturales.

8- Refiere al nivel secundario de la escolaridad obligatoria.
} 
dado Biología en el secundario pero no es profesor"9 (Entrevista a Claudio, 21/12/2009). Con ello, no lo reconocía como par.

Los modos de nombrar a ese otro llevan marcas de prestigio vinculadas a los diferentes grados de especialización que las personas portan en relación con el área de las Ciencias Naturales y en función de su inscripción en el área de primaria o de programas. La normativa estatal es reinterpretada por los actores en la cotidianeidad escolar (Rockwell, 1989). Siguiendo a Maroy y Dupriez (2000), se produce un juego donde la regulación de las escuelas no supone una direccionalidad de arriba abajo ni la posibilidad de asir su cotidianeidad únicamente a partir de lo dispuesto en las resoluciones.

La producción de un cuerpo especializado de personas, maestros de grado, a cargo de la enseñanza de un conjunto de saberes a un cuerpo de legos e infantes ha sido una de las características constitutivas de la escuela moderna en tanto espacio simbólico y social (QUERRIEN, 1979; TRILLA, 1999). Convertidos en los únicos agentes de Estado a cargo de la enseñanza en el nivel primario, estos actores estatales en la escuela corporizaban al Estado moderno mientras que éste los investía de su autoridad (DUBET, 2005; GIOVINE, 2012). En el orden escolar, se trataba de dos posiciones muy claras y definidas: unos estaban para enseñar y otros para aprender. Estas posiciones relativas y su inscripción dentro de una trama de relaciones vinculada al saber permiten otorgar visibilidad a la conmoción de su configuración histórica. No solamente se trataba de dos polos distinguidos por su relación con el conocimiento y su dominio sino que uno de esos polos, el enseñante, era equivalente de maestro de grado, es decir, denotaba la realización de un trabajo en determinado nivel de escolaridad: la primaria.

En la actualidad, nuevas personas irrumpen en esta histórica relación a partir de su ingreso a las escuelas a través del mismo Estado que sostuvo y promovió la relación anterior y comparten con los maestros de grado el estar a cargo del enseñar.

Con la incorporación de los especialistas en las escuelas circulan diferentes modos de nombrar los puestos de trabajo vinculados a la tarea de enseñar ciencias. Esta diferenciación entre los encargados de la enseñanza puede ser comprendida a partir del concepto de autoridad pedagógica de Bourdieu y Passeron (1998), donde el disimulo de las relaciones de fuerza supone una selección arbitraria de significados pertenecientes a un grupo -el prestigio de la especialización- que se naturaliza, no sin conflictos, al interior de las escuelas. Con el ingreso de los nuevos actores estatales y el reconocimiento de su especialización, los maestros de grado no son reconocidos -y no se reconocen a sí mismos- por los saberes vinculados al campo de conocimiento de las Ciencias Naturales.

\section{Distribución de tiempos y espacios}

Con el funcionamiento de los programas en las escuelas se conmueve, también, la configuración de los tiempos, de los espacios de trabajo y de las personas que están a cargo de éstos. Los encargados especialistas cuentan con tiempos claramente delimitados

9- Esta diferenciación motiva evitar asumir que todos los actores considerados docentes desde el punto de vista de la normativa lo sean desde el punto de vista de los significados que se le atribuyen a tal función. De allí, la necesidad de referir a maestros y especialistas como personas encargadas de la enseñanza. 
para dar clase y señalan que el detenimiento en un mismo contenido o proyecto de enseñanza no es el mismo para los programas que para la escuela común.

Los docentes que ingresaban a las escuelas a través de los programas disponen de uso más flexible y sin apremios por cumplir con una planificación anual. En cambio, para los maestros de grado, la localización del horario de sus clases de ciencias no resulta tan delimitada ni estable dentro de la caja horaria semanal. Los días y los horarios pueden rotar y negociarse con los maestros paralelos y con las otras asignaturas que los maestros tienen que enseñar.

En la escuela $n^{\circ} 8$, las clases de Ciencias Naturales para séptimo B podían desarrollarse en diferentes horarios. En general, Ana contaba con dos bloques de ochenta minutos al final de la mañana que podían mudarse si se intercambiaba las horas con su paralela ${ }^{10}$. El turno mañana iniciaba a las 8 y finalizaba a las 12:15 hs. Ana distribuía este tiempo entre las dos secciones, A y B, y entre las dos áreas, Matemática y Ciencias Naturales. Sus clases se desarrollaban en el aula. Si bien el profesor de ciencias y el laboratorio estaban dentro de la misma escuela, no configuraban parte del universo de posibilidades con las cuales Ana contaba para realizar su trabajo.

Ana planteaba que enseñaba Matemática en detrimento de las Ciencias Naturales y que no le alcanzaban los tiempos para este último campo de conocimiento. Al principio, presté poca atención cuando la escuchaba. Lo que ella decía estaba en línea con lo planteado por la mayoría de los documentos que acompañan las políticas educativas y las voces de algunos especialistas de la didáctica específica cuando señalaban que los maestros de grado dedican poco tiempo a la enseñanza del área. Cuestiones que suelen ser atribuidas a su floja formación y a enfoques tradicionales de enseñanza ${ }^{11}$ donde "[...] aparece la típica germinación del poroto o el sistema solar con sus planetas coloridos" (UNESC0, 2005, p. 2).

Sin embargo, lo que sí me sorprendió fueron los indicios que iban llegando junto a mis anotaciones y registros. Al finalizar el trabajo de campo, había asistido a más de veinte clases de Ciencias Naturales a cargo de Ana con una duración de ochenta minutos cada una. El tiempo dedicado a la enseñanza del área frente a los alumnos involucraba un promedio de tres horas de clase semanales. El diseño curricular vigente para la Ciudad de Buenos Aires no establece la cantidad de horas que se debe enseñar Ciencias Naturales (BUENOS AIRES, 2004). Durante mi presencia en ésta y otras escuelas pude notar que en los cronogramas semanales correspondientes al segundo ciclo de jornada simple figuran tres horas de clase semanales y Ana cumplía -en promedio- con esta carga horaria. Lo llamativo era que ella no lo percibiera de este modo.

Las clases de Luciano se desarrollaban en el laboratorio de la escuela de 9:30 a 11:30 hs durante el turno mañana. Su horario de trabajo dentro de la escuela coincidía con el de los maestros de grado, aunque al estar en el laboratorio prácticamente no se cruzaba con ellos. Luciano señalaba que el uso del tiempo era diferente del que se hacía en las clases de Ciencias Naturales en el aula. En Club de Ciencias, podían "explorar más a fondo",

10- Es una categoría nativa utilizada en las escuelas para designar a un maestro que enseña en el mismo grado que otro pero en diferente sección. 11- Esta caracterización puede consultarse en documentos correspondientes a la política educativa local, a organizaciones internacionales y revistas de la didáctica específica en español. 
tratar de que el chico pensara las situaciones y que el conocimiento se lograra a partir de preguntas que él mismo se elaborara (Entrevista a Luciano, 26/11/2009). Decía que esto le había implicado una desestructuración importante ya que como maestro de grado corría con los tiempos para poder enseñar.

En la escuela $n^{\circ} 20$, la directora presentaba a la escuela a través de los proyectos en ciencias. El término proyectos aludía a las propuestas de enseñanza que se enmarcaban dentro de los programas que se mostraban públicamente en las muestras de ciencias, no así lo enseñado en Naturales. Con esta última denominación hacía referencia a las clases que estaban a cargo de los maestros de grado dentro de lo que se consideraba el área de nivel primario. El asistente técnico y los maestros también realizaban esta distinción; referían a trabajos que se realizaban en el aula y a otros que se realizan en IAC.

Claudio desarrollaba sus clases en el aula, en la biblioteca o en el parque de la escuela. También señalaba que a él le gustaba realizar experiencias pero temía ir al laboratorio. Decía que no era su espacio y que le daba miedo romper un tubo de ensayo.

Claudio y su paralela -la maestra de séptimo dedicada a las asignaturas de Prácticas del Lenguaje y Ciencias Sociales- se organizaban para dar clases un día cada uno en cada sección, es decir, en séptimo A y séptimo B. A pesar de esta movilidad horaria, los miércoles a la mañana, cuando Dante llegaba, Claudio indicaba a los niños que debían ir al laboratorio porque tenian IAC. Este horario era fijo. Generalmente, sus clases se desarrollaban en el laboratorio. En relación con los tiempos, Dante expresaba:

El maestro que tiene que dar ciencias de manera normal lo tiene que hacer siguiendo los temas y los contenidos que están en la planificación anual [...] En IAC nos importa que haya conceptos básicos y explotar al máximo eso que al chico le interesó en ese momento [...] y capaz que lo que para la planificación anual sería una pérdida de tiempo, para IAC no. (Entrevista a Dante, 22/12/2009).

Naturales y dar ciencias de manera normal se diferenciaba de IAC. Todo ocurría dentro de la misma escuela. Cuando Dante trabajaba con séptimo en el laboratorio, Claudio podía quedarse a corregir carpetas o preparar sus próximas clases en el aula. Creía que él estaba “[...] más que nada para control docente, no para aportar algo en la clase" (Entrevista a Claudio, 21/12/2009).

La percepción de espacios diferenciados interpretados como espacios físicos -aula y laboratorio- o bien como asignaturas o bien como proyectos inciden en lo que los actores interpretan como espacios de pertenencia y no pertenencia, a los que tienen o no tienen acceso. Del mismo modo, opera la percepción de los tiempos como insuficientes cuando los maestros de grado efectivamente cumplen con las horas de enseñanza previstas para el área, o bien cuando los nuevos actores los interpretan como una posibilidad de profundización donde disponen del tiempo para ampliar el interés sin necesidad de cumplir con temarios o de enseñar de manera común. Todo ello puede pensarse como constitutivo de la lógica práctica (BOURDIEU, 2007) del orden escolar (MILSTEIN; MENDES 1999, 2013) y opera no de modo ajeno a la especificidad de los 
procesos de enseñanza y aprendizaje de un campo de conocimiento que se desarrollan en la escuela.

\section{Valoraciones del trabajo docente}

A la peculiar diferenciación que se producía en los modos de nombrar los puestos de trabajo, los usos de los tiempos y los espacios para la enseñanza de las ciencias al interior de las escuelas, se sumaba una valoración negativa sobre el trabajo desempeñado por los maestros de grado. Esos modos de calificar su trabajo, no sólo formaban parte de algunas caracterizaciones realizadas por una parte de los especialistas de la didáctica y de algunos lineamientos que argumentaban el desarrollo de políticas educativas, sino que también había ingresado a la escuela y era asumida por los padres de algunos alumnos.

En la escuela $n^{\circ} 8$, la directora decía que cuando asumió la conducción, la escuela estaba desorganizada y que los maestros tenían miedo. En los inicios de su trabajo, unos padres fueron a decirle que "una maestra enseñaba mal" y que querían hacer un acta. "Se acercaron patoteando". La directora les dijo que la hicieran, que su hijo era abogado y les iba a iniciar y ganar una demanda por calumnias y perjuicios. Parece que los padres nunca hicieron el acta y que el cuerpo docente valoró mucho su actitud. “¿Qué cosas tan graves pueden hacer los docentes?”, me preguntaba, “¿equivocarse en un boletín? A menos que sea grave, las cosas después se ven puertas para adentro" (Notas de campo, 10/08/2009).

La cadencia de estas preguntas y su mirada sostenida al enunciarlas me llevaron a invertir la pregunta, no ya en términos de lo mirado (la escuela) sino de quiénes y cómo miran -miramos- la escuela. ¿Cómo podía ser que de pronto los maestros no supieran nada? ¿Qué era aquello que había cambiado para que ahora se considerara que los docentes enseñaban mal?

A diferencia de los padres, los maestros planteaban tener que ocuparse de "cosas súper básicas”. Una mañana, la maestra de quinto grado comentaba a sus colegas que la mamá de un alumno que había vuelto a la escuela después de haber faltado por varios días le había dicho: “[...] acá te lo traigo de vuelta, ponémelo al día con lo que hicieron”. La maestra, enojada, se preguntaba dónde había quedado el pedirle la tarea al compañero. Desde la perspectiva de la madre, llevar a su hijo a la escuela para que hiciera las tareas atrasadas y siguiera al día con las actuales era una responsabilidad de la docente y no de la familia. En el mismo diálogo, Ana se preguntaba: “¿cómo quieren que nos preocupemos cómo enseñar si hay tantas cosas anteriores que resolver como éstas?”. Aclaraba que no se trata de que no fuera importante preocuparse por enseñar bien, al contrario, consideraba que había que mejorar, pero para ella y su colega quedaba "tan en último lugar..." (Notas de campo, 25/06/2009).

¿Qué significaba enseñar mal y bien? La directora defendía a los maestros frente a los padres que se quejaban a la par que destacaba el trabajo realizado por Luciano, precisamente, por su especialización. Este reconocimiento otorgado por la directora resultaba relativo a sus interlocutores. Frente a los padres, la directora defendía a los maestros y si algo no funcionaba se solucionaba puertas adentro. Pero puertas adentro, se esperaba que los maestros consultaran al profesor especializado. 
En la escuela $n^{\circ} 20$, el miedo de uno de los maestros fue explicitado a propósito de mi presencia en la escuela. Durante una de mis visitas me encontré con Claudio junto a su esposa dentro del predio de la escuela. Al verlos a los dos juntos les mencioné que creía conocerlos. Finalmente pudimos reponer la conexión. Los había visto bailando salsa en un cumpleaños. Esto pareció aliviar al maestro.

Más tarde, Claudio le comentó a su maestra paralela que me conocía. A lo que ella respondió: “¡Viste que no tenías que tener miedo!”. Después de este intercambio, el maestro me aclaró que sus clases no eran "lo de IAC", "eso es otra cosa" (Notas de campo, 09/09/2009). Lo suyo y lo de Dante eran dos cosas diferentes.

Lo hasta aquí expuesto permite identificar que los significados del enseñar bien y el enseñar mal también se encuentran en relación con los puestos de trabajo, sus denominaciones, el uso de los tiempos y de los espacios dentro de la escuela.

\section{Re(ordenamiento) de la vida escolar: la conmoción de un vínculo con el saber}

El reordenamiento que se produce en el espacio escolar no se explica por una nueva distribución de actores y elementos que por el solo hecho de ingresar se suman a un orden escolar establecido. Se trata de un reordenamiento de reglas y modos implícitos de decir y estar en las escuelas. Esto supone desplazamientos en los modos de habitarlas que se actualizan ante la llegada de nuevas personas encargadas de la enseñanza que hacen uso de recursos tales como el laboratorio.

Estos modos involucran valoraciones, sentimientos, percepciones sobre los diferentes grados de especialización que los encargados de la enseñanza detentan en el espacio escolar y que resultan cualificados a partir de sus denominaciones y de referencias en términos de enseñar bien y enseñar mal, tiempos flexibles para profundizar y tiempos que no alcanzan, el aula y el laboratorio. En la medida que se actualizan los modos de percibir se interiorizan clasificaciones del espacio social de la escuela. De este modo, el laboratorio al igual que el puesto de trabajo de las personas que trabajaban en los programas gozaban de un prestigio que ni los maestros de séptimo grado ni su espacio de trabajo en el aula tenían cuando se realizaba la misma tarea: enseñar Ciencias Naturales.

Estos reordenamientos permiten reconstruir los efectos de Estado en el impacto que produce la redistribución de personas y elementos dentro de las escuelas y disposiciones a actuar que se interiorizan. En la medida que se interiorizan tienden a sustancializarse las propiedades, abstrayendo el escenario en el cual se inscriben discursos y prácticas, que resultan relativos a otros.

La eficacia de esta abstracción reside en el reconocimiento de dicho prestigio por parte de los maestros de grados y de un desconocimiento -en términos de Bourdieu y Passeron (1998) - del efecto que este modo de clasificar tiene en las propias maneras de significar el espacio escolar, las prácticas de enseñanza, los espacios y los tiempos.

Lo interesante de esta regla implícita que opera en la escuela, donde Ana pide que no la compare con Luciano, es su eficacia simbólica, ya que Ana y Luciano no habían trabajado juntos. Tan sólo conocían quién era cada uno, qué función tenían dentro de la 
escuela y compartido unos cuarenta minutos reloj en oportunidad de que Luciano invitara a los alumnos de Ana a participar del Club de Ciencias. De esta manera, ¿cómo ponderar si el profesor de ciencias realmente enseña bien sin haber trabajado con él, sin conocer el trabajo educativo que realizaba en el laboratorio, sin conocer su enfoque didáctico?

De manera semejante, en la escuela $n^{\circ} 20$, un día se acercó el otro maestro de tercer grado -el que no estudiaba ingeniería agrónoma-, me preguntó si yo pertenecía a un programa y si el año próximo sería capacitadora: “[...] yo los necesito, no los voy a rechazar, porque ahora tengo a mi paralelo que es una maravilla, pero el año que viene él se va" (Notas de campo, 03/11/2009). Dentro de la escuela, el estudiante de ingeniería agrónoma se convertía en un especialista; para el equipo de conducción, en un ingeniero agrónomo recibido; y para su paralelo en una maravilla. Este maestro demandaba como necesaria su especialización. Esta necesariedad se vuelve sintomática de la homologación que vincula la buena enseñanza con la especialización de los conocimientos y los programas. Al igual que en la escuela $n^{\circ} 8$, ¿cómo saber que se va a necesitar de otra persona para trabajar enseñando Ciencias Naturales sin conocerla? ¿Tan solo el ingreso a la escuela a través de un puesto de trabajo vinculado a un programa se vuelve garantía de buena enseñanza?

La primera hipótesis de trabajo es que el sistema de enseñanza está atravesado por personas que también están encargadas de enseñar Ciencias Naturales en las escuelas que ingresan como nuevas presencias organizadas desde el propio Estado. Los efectos de este ingreso pueden reconstruirse a partir de un proceso de diferenciación en las denominaciones de los puestos de trabajo, el uso de tiempos y espacios, y las connotaciones de valor que acompañan a los elementos anteriores.

La segunda hipótesis que sostengo es que dicho ingreso produjo desconcierto al irrumpir en modos de ordenamiento de la vida cotidiana escolar en la cual los maestros de grado eran quienes detentaban el lugar de enseñante en la escuela primaria y eran reconocidos tanto por las autoridades estatales como por los alumnos y las familias. Indicios de este desconcierto pueden encontrarse en los vínculos con las familias de los alumnos, en la resistencia que los asistentes técnicos percibían en los maestros, entre otros.

En este esquema, los posicionamientos relativos de los encargados de la enseñanza, los espacios, los tiempos, las valoraciones y connotaciones en torno a los elementos anteriores, los modos de regulación de los trabajos de las diferentes áreas educativas y el carácter desde el cual el Estado reviste de autoridad a los maestros y a los docentes especialistas, presentan un punto de contacto con lo que Bourdieu y Passeron (1998) denominaron como arbitrario cultural. Estas categorías permiten destacar que no sólo se trata de elementos, configuraciones, dispositivos o formatos sino de significados inherentes a estos elementos y configuraciones. Asimismo, permiten advertir que no se trata de analizar solo las propuestas didácticas, las concepciones de los alumnos y docentes vinculados a los saberes disciplinares o las interacciones que se producen en clase a propósito del conocimiento, se trata de que junto a esto se imponen significados que son constitutivos del orden escolar. Los posicionamientos relativos de los encargados de la enseñanza y su distribución al interior de las escuelas afectan y modulan las relaciones que se propician en relación con los saberes de un campo de conocimiento. 
Desde esta lectura, intentar comprender los espacios de trabajo de Ciencias Naturales correspondientes al área de primaria únicamente desde lentes teóricas surgidas en el campo de la didáctica específica no permite ponderar que la escuela y la cultura legítima a ser transmitida -el arbitrario cultural- no se vinculaba a la lógica de la especialización de saberes disciplinares. Tampoco permite visibilizar que la autoridad pedagógica de los maestros devenía de su envestidura estatal cuya función consistía en actuar sobre la infancia y sus familias.

El principal efecto del ingreso de los docentes especialistas reside en cierto desdibujamiento de la autoridad pedagógica que portan los maestros de grado. Dicha autoridad se sostenía por ser considerados funcionarios del Estado y, como tales, gozar de las atribuciones necesarias para intervenir sobre la infancia. Bajo esta envestidura, recibían el reconocimiento por parte de las familias que aceptaban en la misma operación por la cual enviaban a los niños a la escuela convertirse en objeto de intervención estatal.

Este desdibujamiento se materializa en el cuestionamiento del trabajo realizado por los maestros respecto de la enseñanza de las ciencias; en directivos que puertas adentro persiguen que los maestros apelen a los docentes especializados y que puertas afuera, para quienes se acercan a conocer las escuelas, las presentan a través de sus proyectos de ciencias y de sus especialistas; en padres dispuestos a elaborar actas en las cuales quede explicitado que los maestros enseñan mal; y en pedidos que las familias realizan a las maestras respecto de cómo deben actuar cuando un niño falta a la escuela. Las madres pasan a encargarles a las maestras que pongan a sus hijos al día con los contenidos y tareas realizadas cuando antes eran las maestras quienes les pedían a las madres que se encargaran de que los niños copiaran la carpeta de un compañero. Con este pedido, las maestras organizaban facetas de la vida doméstica de las familias en base al trabajo de la escuela. Ahora, eran las madres quienes pugnaban por organizar la vida escolar a partir de decirle a las maestras cómo debían actuar frente a sus hijos.

Lo inédito de este desdibujamiento de la autoridad pedagógica de los maestros es que resulta relativa al ingreso de otras personas que exhiben autoridad otorgada por el mismo Estado. Estas otras personas ingresan a las escuelas a partir de haber sido entrevistadas por coordinadores que trabajan en programas de ciencias que las aceptaron para trabajar, no ya por puntaje en base a cursos realizados y antigüedad docente, sino en base al reconocimiento en un campo de saber. Los nuevos actores ingresan a las escuelas exhibiendo su envestidura también estatal con la singularidad de que la misma porta, además, el reconocimiento de su especialización.

El desdibujamiento de la autoridad pedagógica de los maestros presenta otra faceta también novedosa en la cual se pasa a concebir que una de las finalidades de la educación de los niños consista en su socialización en campos específicos de conocimiento. La presencia del Estado en la escuela se expande a partir del ingreso de nuevas personas que vienen a otorgar una profundización, un refuerzo, donde la escuela común no llega. Este proceso supone un desplazamiento de los objetivos de la escuela en la formación de la ciudadanía. No se trata de que antaño la función de enseñar campos de conocimiento específicos no formara parte de los objetivos de la escuela sino de la centralidad que adquiere y que amerita que desde las políticas educativas se decida ampliar la presencia 
estatal en la escuela y habilitar el ingreso de personas que bajo los mecanismos históricos por los cuales ingresan los maestros de grado no podrían hacerlo.

Esta eficacia simbólica opera porque tanto los maestros, como quienes ingresan, como algunas familias, han aceptado este nuevo artificio. Esto es que los niños de escuela primaria necesitan acceder a un conocimiento especializado, aunque no se sepa muy bien aún en qué consiste este modo de vincularse con el conocimiento.

\section{Referencias}

BOURDIEU, Pierre. Capital Cultural, escuela y espacio social. Buenos Aires: Siglo XXI, 2012.

BOURDIEU, Pierre. El sentido práctico. Buenos Aires: Siglo XXI, 2007.

BOURDIEU, Pierre; PASSERON, Jean-Claude. La reproducción: elementos para una teoría del sistema de enseñanza. México, DC: Fontanara, 1998.

BOURDIEU, Pierre. Sociología y cultura. México, DC: Grijalbo, 1990.

BUENOS AIRES. Gobierno de la Ciudad de Buenos Aires. Ley $n^{\circ} 3623$, sancionada y promulgada de hecho en 2010. Boletín Oficial de la Ciudad de Buenos Aires, n. 3579, 2011. Disponible en: <http://www. buenosaires.gob.ar/ areas/educacion/lua/pdf/programas_socioeducativos_sept_2013.pdf>. Acceso en: 24 jul. 2015.

BUENOS AIRES. Gobierno de la Ciudad de Buenos Aires. Diseño curricular para la escuela primaria: segundo ciclo de la escuela primaria: educación general básica. Tomo 2. Buenos Aires: Secretaría de Educación. Dirección General de Planeamiento, Dirección de Currícula, 2004.

BUENOS AIRES. Gobierno de la Ciudad de Buenos Aires. Estatuto del docente: ordenanza n 40593 y sus modificatorias. Buenos Aires: Ministerio de Educación de la Ciudad de Buenos Aires, 2014. Disponible en: <http://www.buenosaires.gob.ar>. Acceso en: 24 jul. 2015.

CHARLOT, Bernard. La relación con el saber: elementos para una teoría. Buenos Aires: Libros del Zorzal, 2007.

DUBET, François. ¿Mutaciones institucionales y/0 neoliberalismo? Revista Colombiana de Sociología, Bogotá, v. 25, p. 63-80, 2005.

GEERTZ, Clifford. Descripción densa: hacia una teoría interpretativa de la cultura. In: GEERTZ, Clifford. La interpretación de las culturas. Barcelona: Gedisa, 1992. p. 19-40.

GIOVINE, Renata. El arte de gobernar el sistema educativo: discursos de estado y redes de integración socioeducativas. Buenos Aires: Universidad Nacional de Quilmes, 2012.

GUBER, Rosana. El salvaje metropolitano: reconstrucción del conocimiento social en el trabajo de campo. Buenos Aires: Paidós, 2008. 
MAROY, Christian; DUPRIEZ, Vincent. La régulation dans les systèmes scolaires: proposition théorique et analyse du cadre structurel en Belgique francophone. Revue Française de Pédagogie, Lyon, v. 130, p. 7387, janv./mars. 2000.

MILSTEIN, Diana; MENDES, Héctor. Cuerpo y escuela: dimensiones de la política. Revista Iberoamericana de Educación, Madrid, v. 62, p. 143-161, 2013.

MILSTEIN, Diana; MENDES, Héctor. La escuela en el cuerpo: estudios sobre el orden escolar y la construcción social de los alumnos en escuelas primarias. Madrid: Miño y Dávila, 1999.

MILSTEIN, Diana. La nación en la escuela. Nuevas y viejas tensiones políticas. Buenos Aires: Miño y Dávila - IDES, 2009.

QUERRIEN, Anne. Trabajos elementales sobre la escuela primaria. Madrid: De la Piqueta, 1979.

ROCKWELL, Elsie. De huellas, bardas y veredas: una historia cotidiana de la escuela. In: ROCKWELL, Elsie; MERCAD0, Ruth (Org.). La escuela, lugar del trabajo docente. México, DC: DIE/Cinvestav/IPN, 1989. p. 9-33.

ROCKWELL, Elsie. La experiencia etnográfica: historia y cultura en los procesos educativos. Buenos Aires: Paidós, 2009.

TRILLA, Jaume. Ensayos sobre la escuela: el espacio social y material de la escuela. Barcelona: Laertes, 1999.

TROUILLOT, Michel-Rolph. The Anthropology of the state in the age of globalization close encounters of the deceptive kind. Current Antropology, Chicago, v. 42, n. 1, p. 125-138, feb. 2001.

UNESCO. IIPE - Instituto Internacional de Planeamineto de la Educación. La enseñanza de las ciencias naturales: desafíos y propuestas. Informes periodísticos para su publicación. Buenos Aires: Unesco, 2005.

Recibido en: 03.02.2017. Revisiones en: 09.05.2017. Aprobado en: 05.07. 2017.

Cecilia Acevedo es doctora en Ciencias Sociales y Humanas por la Universidad Nacional de Quilmes y licenciada en Ciencias de la Educación por la Universidad de Buenos Aires. Es docente en la formación pedagógica de los profesorados de ciencias en la Universidad de Buenos Aires, en la formación de profesores del nivel primario y en cursos de posgrado. 